\title{
Fuzzy Multi-Criteria Decision-Making for Evaluating the performance of mutual funds
}

\author{
Shin-Yun Wang ${ }^{1}$, Cheng-Few Lee ${ }^{2}$, Gwo-Hshiung Tzeng ${ }^{3}$ \\ Keywords: Mutual fund, Fuzzy Multiple Criteria Decision Making (FMCDM), Analytic Hierarchy \\ Process (AHP), Nonadditive fuzzy integral. \\ ${ }^{1}$ Department of International Trade, Chung Kuo Institute of Technology \\ ${ }^{2}$ Department of Finance, Rutgers University, New Brunswick, NJ, USA \\ ${ }^{3}$ Institute of Management of Technology, National Chiao Tung University
}

Summary: This paper describes a fuzzy hierarchical analytic approach to determine the weighting of subjective judgments. In addition, it presents a nonadditive fuzzy integral technique to evaluate a mutual fund case as a fuzzy multi-criteria decision-making (FMCDM) problem. When the investment strategies are evaluated from various aspects, such as market timing, stock selection ability, fund size and team work, it can be regarded as an FMCDM problem. Since investors can not clearly estimate each considered criterion in terms of numerical values for the anticipated alternatives/strategies, fuzziness is considered to be applicable. Consequently, this paper uses triangular fuzzy numbers to establish weights and anticipated achievement values. By ranking fuzzy weights and fuzzy synthetic performance values, we can determine the relative importance of criteria and decide the best strategies. This paper applies what is called a $\lambda$ fuzzy measure and nonadditive fuzzy integral technique to evaluate aquatic investment. In addition, we demonstrate that the nonadditive fuzzy integral is an effective evaluation and appears to be appropriate, especially when the criteria are not independent.

\section{Introduction}

Mutual fund, which has huge market potential, has been gaining momentum in the financial market. The complexities are numerous, and overcoming these complexities to offer successful selections is a mutual fund manager challenge. It is important that the limited amount of investing funds should be efficiently allocated many stocks to evaluate aquatic return so as to reduce its risk and to find the optimal combination of invested stocks out of many feasible stocks for mutual fund manager in the market. The purpose of mutual fund is minimizing the risk in allocating the amount of investing funds to many stocks. In a real problem, because of the limit amount of funds to invest into stocks, the solution of the portfolio selection problem proposed by H.Markowitz (1952) has a tendency to increase the number of stocks selected for mutual fund. In a real investment, a fund manager first makes a decision on how much proportion of the investment should go to the market, and then he invests the funds to stocks. After that, maximizing the mutual fund return is the primary goal of mutual fund manager in a corporation. Usually, the mutual fund return reflects the financial performance of a fund corporation for operating and development. This paper explores which criteria that including the 
market timing; stock selection ability; fund size and team work by taking as overall evaluation and adopting the financial rations as evaluation criteria can lead to high financial performance. The financial performance is evaluated by fuzzy multi-criteria decision-making (FMCDM), this supports managers' decision- making.

The financial statement analysis, which is used to evaluate financial performance, involves ratio analysis, trend analysis. The ratio analysis provide a basis for a company to compare with other companies in the same industry and trend analysis evaluating trends in the company financial position over time. Investment planning and decision-making in mutual fund are essentially conflict analysis characterized by sociopolitical, environmental, and economic value judgments. Several alternatives strategies have to be considered and evaluated in terms of many different criteria resulting in a vast body of data that are often inaccurate or uncertain. Therefore, the purpose of this article is to develop an empirically-based framework for formulating and selecting a mutual fund strategy. We propose a hierarchical Fuzzy Multi-Criteria Decision-Making (FMCDM) framework, where we combine AHP and fuzzy measure methods in order to determine the relative weights of each criterion. The proposed strategies are then ranked using the fuzzy integral method. To demonstrate the validity of this method, one is used as an illustrative case. The results describe the strategies that were adopted by this have proven to be very successful in performance. This also proves the effectiveness of the approach proposed by this paper.

In real world systems, the decision-making problems are very often uncertain or vague in a number of ways. Due to lack of information, the future state of the system might not be known completely. This type of uncertainty has long been handled appropriately by probability theory and statistics. However, in many areas of daily life, such as fund, stock, debt, derivates and others, human judgment, evaluation, and decisions often employ natural language to express thinking and subjective perception. In these natural languages the meaning of words is often vague, the meaning of a word might be well defined, but when using the word as a label for a set, the boundaries within which objects do or do not belong to the set become fuzzy or vague. Furthermore, human judgment of events may be significantly different based on individuals' subjective perceptions or personality, even using the same words. Fuzzy numbers are introduced to appropriately express linguistic variables. We will provide a more clear description of linguistic expression with fuzzy scale in a later section.

In this paper the fuzzy hierarchical analytic approach was used to determine the weights of criteria from subjective judgment, and a nonadditive integral technique was utilized to evaluate the performance of investment strategies for mutual fund. Traditionally, researchers have used additive techniques to evaluate the synthetic performance of each criterion. In this article, we demonstrate that the nonadditive fuzzy integral is a good means of evaluation and appears to be more appropriate, especially when the criteria are not independent situations. The conceptual investment of mutual fund is discussed in the next section, and the fuzzy hierarchical analytic approach and nonadditive fuzzy integral evaluation process for multicriteria decision-making (MCDM) problem are derived in the subsequent section. Then an illustrative example is presented, applying the MCDM methods for aquatic 
investment processors, after which we discuss and show how the MCDM methods in this paper are effective. Finally, the conclusions are presented.

\section{Concept Investment of Mutual Fund}

Recently, mutual fund has been widely discussed. Connor and Korajczyk (1991) developed a method of portfolio performance measurement using a competitive version of the arbitrage pricing theory (APT). However, they ignored any potential market timing by managers. Fama (1972) indicates that there are two ways for fund managers to obtain abnormal returns. The first one is security analysis, which is the ability of fund managers to identify the potential winning securities. The second one is market timing, which is the ability of portfolio managers to time market cycles and take advantage of this ability in trading securities. Lehmann and Modest (1987) combined the APT performance evaluation method with the Treynor and Mazuy (1966) quadratic regression technique. They found statically significant measured abnormal timing and selectivity performance by mutual funds. They also examined the impact of alternative benchmarks on the performance of mutual funds finding that performance measures are quite sensitive to the benchmark chosen and finding that a large number of negative selectivity measures. Also, Henriksson (1984) found a negative correlation between the measures of stock selection ability and market timing.

Fundamental mutual fund performance architecture includes four components as market timing; stock selectivity ability; fund size and team work. An empirical investigation discusses conceptual and econometric issues associated with identifying four components of mutual fund performance, the empirical results obtained using the technique developed by Bhattacharya and Pfleiderer (1983) indicate that at the individual level there is some evidence of superior forecasting ability on the part of the fund manager. This result has an important implication. Mutual fund manager with no forecasting skill might consider a totally passive management strategy and just provide a diversification service to their investor. Therefore, from a practical point of view, we adopt four aspects to evaluate the performance of mutual fund.

\section{The Method of Fuzzy Multi-Criteria Decision-Making}

Traditional AHP is assumed that there is no interaction between any two criteria within the same hierarchy. However, a criterion is inevitably correlated to another one with the degrees in reality. In 1974, Sugeno introduced the concept of fuzzy measure and fuzzy integral, generalizing the usual definition of a measure by replacing the usual additive property with a weak requirement, i.e. the monotonic property with respect to set inclusion. In this section, we give a brief to some notions from the theory of fuzzy measure and fuzzy integral.

\subsection{General fuzzy measure}

The fuzzy measure is a measure for representing the membership degree of an object in candidate sets. It assigns a value to each crisp set in the universal set and signifies the degree of evidence or belief of 
that element's membership in the set. Let $X$ be a universal set. A fuzzy measure is then defined by the following function $g: \aleph \rightarrow[0,1]$

That assigns each crisp subset of $X$ a number in the unit interval $[0,1]$. The definition of function $g$ is the power set $\aleph$. When a number is assigned to a subset $A \in \aleph, g(A)$, this represents the degree of available evidence or the subject's belief that a given element in $X$ belongs to the subset $A$. This particular element is most likely found in the subset assigned the highest value.

In order to quantify a fuzzy measure, function $g$ needs to conform to several properties. Normally function $g$ is assumed to meet the axiom of the probability theory, which is a probability theory measurement. Nevertheless, actual practice sometimes produces a result against the assumption. This is why the fuzzy measure should be defined by weaker axioms. The probability measure will also become a special type of fuzzy measure. The axioms of the fuzzy measures include:

(1) $g(\phi)=0, \quad g(X)=1$ (boundary conditions);

(2) $\forall A, B \in \aleph$, if $A \subseteq B$ then $g(A) \leq g(B)$ (monotonicity).

Once the universal set is infinite, it is required to add continuous axioms (Klir and Folger, 1998) .

Certainly the elements in question are not within the empty set but within the universal set, regardless of the amount of evidence from the boundary conditions in Axiom 1.

The fuzzy measure is often defined with an even more general function:

$g: \beta \rightarrow[0,1]$

where $\beta \subset \aleph$ so that:

1. $\phi \in \beta$ and $X \in \beta$;

2. if $A \in \beta$, then $\bar{A} \in \beta$

3. $\beta$ is closed under the operation of set function; i.e., if $A \in \beta$ and $B \in \beta$, then $A \cup B \in \beta$.

The set $\beta$ is usually called the Borel field. The triple $(X, \beta, g)$ is called a fuzzy measure space if $g$ is a fuzzy measure on a measurable space $(X, \beta)$.

It is sufficient to consider the finite set in actual practice. Let $X$ be a finite criterion set, $X=\left\{x_{1}, x_{2}, . ., x_{n}\right\}$ and the power set $\aleph$ be a class of all of the subsets of $X$. It can be noted that $g\left(\left\{x_{i}\right\}\right)$ for a subset with a single element, $x_{i}$ is called a fuzzy density. In the following paragraph, we use $g_{i}$ to represent: $g\left(\left\{x_{i}\right\}\right)$.

The term "general fuzzy measure" is used to designate a fuzzy measure that is only required to satisfy the boundary condition and monotonic to differentiate the $\lambda$-fuzzy measure, $F$-additive measure, and classical probability measure.

\section{$3.2 \lambda$ - Fuzzy measure}

The specification for general fuzzy measures requires the values of a fuzzy measure for all subsets in $X$. Sugeno has developed the $\lambda$-additive axiom (Sugeno and Terano, 1997) in order to reduce the difficulty of collecting information. Let $(X, \beta, g)$ be a fuzzy measure space: $\lambda \in(-1, \infty)$. if $A \in \beta$, $B \in \beta$; and $A \cap B=\phi$, and

$$
g(A \cup B)=g(A)+g(B)+\lambda g(A) g(B)
$$

If this holds, then fuzzy measure $g$ is $\lambda$-additive. This kind of fuzzy measure is named $\lambda$ fuzzy 
measure, or the Sugeno measure. In this paper we denote this $\lambda$-fuzzy measure by $g_{i}$ to differentiate from other fuzzy measures. Based on the axioms above, the $\lambda$-fuzzy measure of the finite set can be derived from fuzzy densities, as indicated in the following equation:

$$
g_{\lambda}\left(\left\{x_{1}, x_{2}\right\}\right)=g_{1}+g_{2}+\lambda g_{1} g_{2}
$$

where $g_{1}, g_{2}$ represents the fuzzy density.

Let set $X=\left\{x_{1}, x_{2}, \ldots, x_{n}\right\}$ and the density of fuzzy measure $g_{i}=g_{\lambda}\left(\left\{x_{i}\right\}\right)$, which can be formulated as follows:

$$
g_{\lambda}\left(\left\{x_{1}, x_{2}, \ldots, x_{n}\right\}\right)=\sum_{i=1}^{n} g_{i}+\lambda \sum_{i_{1}=1}^{n-1} \sum_{i_{2}=i_{1}+1}^{n} g_{i_{1}} g_{i_{2}}+\cdots+\lambda^{n-1} g_{1} g_{2} \cdots g_{n}
$$

For an evaluation case with two criteria, $A$ and $B$, there are three cases based on the above properties.

Case 1: if $\lambda>0$, i.e. $g_{\lambda}(A \cup B)>g_{\lambda}(A)+g_{\lambda}(B)$, implying that $A$ and $B$ have a multiplicative effect.

Case 2: if $\lambda=0$, i.e. $g_{\lambda}(A \cup B)=g_{\lambda}(A)+g_{\lambda}(B)$, implying that $A$ and $B$ have an additive effect.

Case 3: if $\lambda<0$, i.e. $g_{\lambda}(A \cup B)<g_{\lambda}(A)+g_{\lambda}(B)$, implying that $A$ and $B$ have a substitutive effect.

The fuzzy measure is often used with the fuzzy integral for aggregating information evaluation by considering the influence of the substitutive and multiplication effect among all criteria.

\subsection{Fuzzy integral (Sugeno and Terano, 1997; Sugeno, 1974; Sugeno and Kwon, 1995)}

In a fuzzy measure space $(X, \beta, g)$, let $h$ be a measurable set function defined in the fuzzy measurable space. Then the definition of the fuzzy integral of $h$ over $A$ with respect to $g$ is

$$
\int_{A} h(x) d g=\sup _{\alpha \in[0,1]}\left[\alpha \wedge g\left(A \cap H_{\alpha}\right)\right.
$$

where $H_{\alpha}=\{x \mid h(\mathrm{x}) \geq \alpha \quad\} . A$ is the domain of the fuzzy integral. When $A=X$, then $A$ can be taken out. Next, the fuzzy integral calculation is described in the following. For the sake of simplification, consider a fuzzy measure $g$ of $(X, \aleph)$ where $X$ is a finite set. Let $h: x \rightarrow[0,1]$ and assume without loss of generality that the function $h\left(x_{j}\right)$ is monotonically decreasing with respect to $j$, i.e., $h\left(x_{1}\right) \geq h\left(x_{2}\right) \geq \cdots \geq h\left(x_{n}\right)$. To achieve this, the elements in $X$ can be renumbered. With this, we then have

$$
\int h(x) d g=\vee_{i=1}^{n}\left[f\left(x_{i}\right) \wedge g\left(x_{i}\right)\right]
$$

where $X_{i}=\left\{x_{1}, x_{2}, \cdots, x_{i}\right\}, i=1,2, \cdots, n$.

In practice, $h$ is the evaluated performance on a particular criterion for the alternatives, and $g$ represents the weight of each criterion. The fuzzy integral of $h$ with respect to $g$ gives the overall evaluation of the alternative. In addition, we can use the same fuzzy measure using Choquet's integral, defined as follows (Murofushi and Sugeno, 1991).

$$
\int h d g=h\left(x_{n}\right) g\left(X_{n}\right)+\left[h\left(x_{n-1}\right)-h\left(x_{n}\right)\right] g\left(X_{n-1}\right)+\cdots+\left[h\left(x_{1}\right)-h\left(x_{2}\right)\right] g\left(X_{1}\right)
$$

The fuzzy integral model can be used in a nonlinear situation since it does not need to assume the 
independence of each criterion.

\subsection{Fuzzy integral multi-criteria assessment methodology}

The fuzzy integral is used in this study to combine assessments primarily because this model does not need to assume independence among the criteria. The fuzzy integral proposed by Sugeno (1974) and Sugeno and Kwon (1995) is then applied to combine the efficiency value of those related criteria to produce a new combined performance value. A brief overview of the fuzzy integral is presented here: Assume under general conditions, $h\left(x_{1}^{k}\right) \geq \cdots \geq h\left(x_{i}^{k}\right) \geq \cdots \geq h\left(x_{n}^{k}\right)$, where $h\left(x_{i}^{k}\right)$ is the performance value of the $k$-th alternative for the $i$ th criterion, the fuzzy integral of the fuzzy measure $g(\bullet)$ with respect to $h(\bullet)$ on $\aleph(g: \aleph \rightarrow[0,1])$ can be defined as follows. (Cheng and Tzeng, 2001; Chiou and Tzeng, 2002; Keeney and Faiffa, 1976]

(c) $\int^{k} h d g=h\left(x_{n}^{k}\right) g_{\lambda}\left(X_{n}^{k}\right)+\left[h\left(x_{n-1}^{k}\right)-h\left(x_{n}^{k}\right)\right] g_{\lambda}\left(X_{n-1}^{k}\right)+\cdots+\left[h\left(x_{1}^{k}\right)-h\left(x_{2}^{k}\right)\right] g_{\lambda}\left(X_{1}^{k}\right)$

where, $g_{\lambda}\left(X_{1}^{k}\right)=g_{\lambda}\left(\left\{x_{1}^{k}\right\}\right), g_{\lambda}\left(X_{2}^{k}\right)=g_{\lambda}\left(\left\{x_{1}^{k}, x_{2}^{k}\right\}\right), \ldots, g_{\lambda}\left(X_{n}^{k}\right)=g_{\lambda}\left(\left\{x_{1}^{k}, x_{2}^{k}, \cdots, x_{n}^{k}\right\}\right)$

The fuzzy measure of each individual criterion group $g_{\lambda}\left(X_{n}^{k}\right)$ can be expressed $\sum_{i=1}^{n} g_{\lambda}\left(x_{i}^{k}\right)+\lambda \sum \sum g_{\lambda}\left(\left\{x_{i}\right\}\right) g_{\lambda}\left(\left\{x_{j}\right\}\right)+\cdots \lambda^{n-1} g_{\lambda}\left(\left\{x_{1}\right\}\right) \cdots g_{\lambda}\left(\left\{x_{n}\right\}\right)$ as follows:

$g_{\lambda}\left(X_{n}^{k}\right)=g_{\lambda}\left(\left\{x_{1}^{k}, x_{2}^{k} \cdots x_{n}^{k}\right\}\right)$

$=\sum_{i=1}^{n} g_{\lambda}\left(x_{i}^{k}\right)+\lambda \sum \sum g_{\lambda}\left(\left\{x_{i}\right\}\right) g_{\lambda}\left(\left\{x_{j}\right\}\right)+\cdots \lambda^{n-1} g_{\lambda}\left(\left\{x_{1}\right\}\right) \cdots g_{\lambda}\left(\left\{x_{n}\right\}\right)$

$=\frac{1}{\lambda}\left[\prod_{i=1}^{n}\left(1+\lambda g_{\lambda}\left(x_{i}^{k}\right)\right)-1\right] \quad$ for $-1<\lambda<+\infty$

$\lambda$ is the parameter that indicates the relationship among related criteria (if $\lambda=0$, equation (7) is an additive form, if $\lambda \neq 0$, equation (7) is a non-additive form). The fuzzy integral defined by equation (c) $\int f d g$ is called the Choquet integral.

\section{Evaluation Model for Prioritizing the mutual fund strategy}

This study utilized the PATTERN (Planning Assistance Through Technical Evaluation of Relevance Number) method (NASA, 1965, 1966; Tang, 1999; Tzeng, 1977; Tzeng and Shiau, 1987) to build up a hierarchical system for evaluating mutual funds strategies. Its analytical procedures stem from three steps: (1) aspects, (2) issues, and (3) strategies. In this section we focus on scenario writings and building relevance trees. Scenario writing is based on determining the habitual domain (Yu, 1985, 1990, 1995) , i.e., past problem understanding, personal experience, knowledge, and information derived from brainstorming techniques so as to determine the factors affecting the successful selection of mutual funds capability. We consider the problems from four aspects: (1) Market timing; (2) Stock selection ability; (3) fund size (4) team work. And, the mutual funds with investment style classified as S1: Asset Allocation style; S2: Aggressive Growth style; S3: Equity Income style; S4: Growth style; S5: 
Growth Income style. Based on a review of the literature, personal experience, and interviews with senior mutual funds managers, relevance trees are used to create hierarchical strategies for developing the optimal selection strategy of building up mutual funds. The elements (nodes) of relevance trees are defined and identified in hierarchical strategies, the combination of which consists of an evaluating mechanism for selecting a mutual fund strategy, as shown in Fig. 1.

\subsection{Evaluating the mutual fund strategy hierarchy system}

Minimum risk or maximum return is usually used as the only measurement index in traditional evaluation methods. Within a dynamic and diversified decision-making environment, this approach may neglect too much valuable information in the process. Hence, we propose a FMCDM method to evaluate the hierarchy system for selecting strategies. In addition, the issues in the investment process are sometimes vague. When this occurs, the investment process becomes ambiguous and subjective for the investor. The evaluation is conducted in an uncertain, fuzzy situation and to what extent vague criteria are realized by research is unknown (Tang and Tzeng 1999;Chiou and Tzeng, 2002). Evaluation in an uncertain, fuzzy situation applies to the formulation of mutual funds strategies as well. We have chosen a fuzzy multiple criteria evaluation method for selecting and prioritizing the mutual fund strategies to optimize the real scenarios faced by manager or investors.

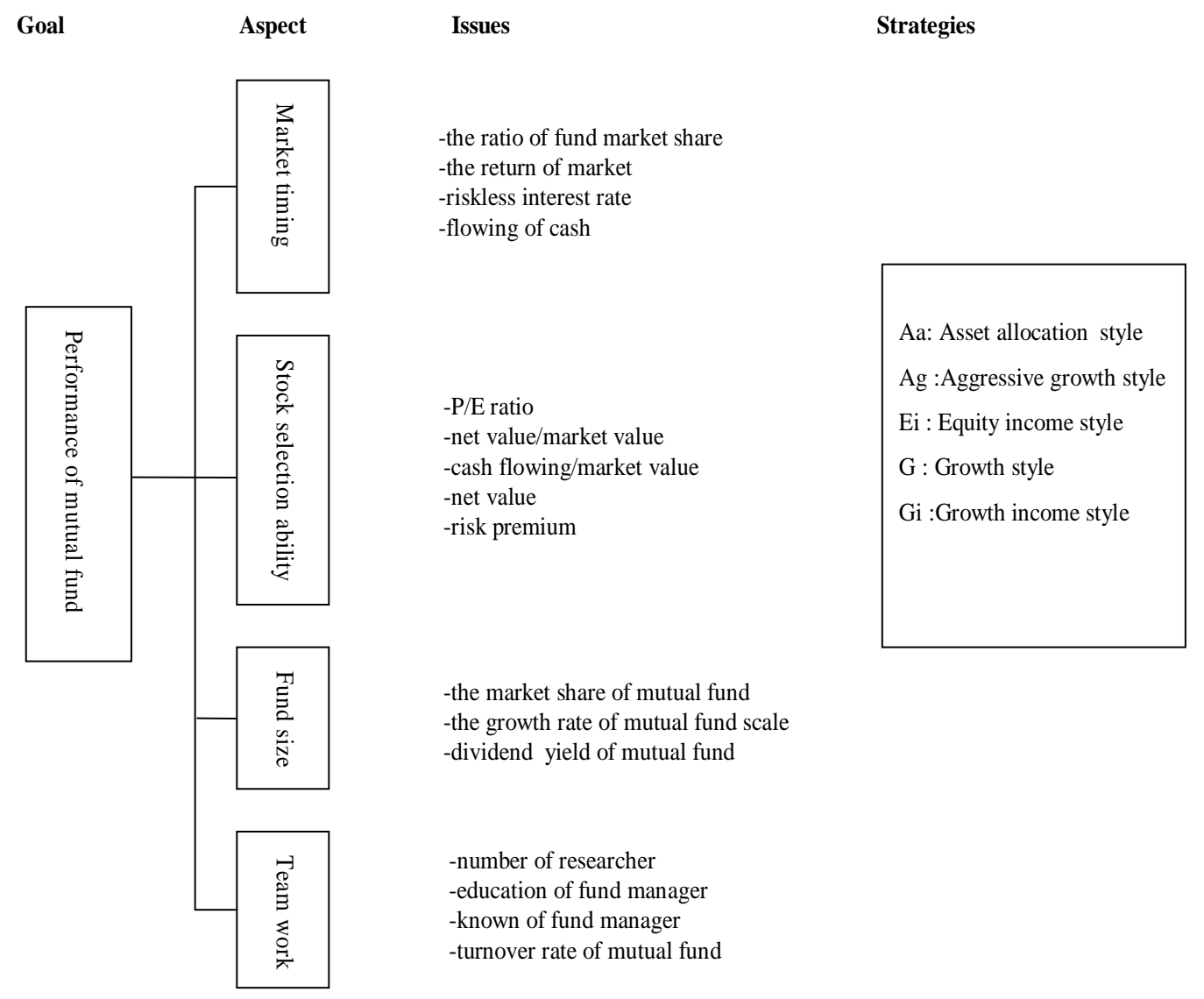

Fig.1 Relevance system of hierarchy tree for evaluating mutual fund strategy 


\subsection{The process for evaluating and prioritizing mutual fund strategies}

Bellman and Zadeh (1970) were the first to study the decision-making problem in a fuzzy environment and initiated FMCDM. In this study, we use this method to evaluate various mutual funds strategies and rank them by their performance. The following subsection describes the method of FMCDM.

\subsubsection{Fuzzy weights for the hierarchy process}

An evaluator always perceives the weight of a hierarchy subjectively. Therefore, to consider the uncertain, interactive effects coming from other criteria when calculating the weight of a specified criterion, we have used fuzzy weights of criteria.

Buckley (1985) was the first to investigate fuzzy weights and fuzzy utility for AHP techniques, extending AHP by geometric means method to derive the fuzzy weights

The fuzzy weights $\tilde{w}_{j}$ corresponding to each criterion is as follows:

$\tilde{w}_{j}=\tilde{r}_{j} \otimes\left(\tilde{r}_{1} \oplus \cdots \oplus \tilde{r}_{m}\right)^{-1}$

where $\tilde{r}_{j}$ is the geometric mean of each row of AHP reciprocal matrix

$\tilde{r}_{j}=\left(\tilde{a}_{j 1} \otimes \cdots \otimes \tilde{a}_{j m}\right)^{1 / m}$

\subsubsection{Measuring criteria}

The evaluators were asked to make subjective judgments using linguistic variable measurement to demonstrate the criteria performance with expressions of effectiveness ranging from "very high", "high”, “fair”, "low”, to "very low”. Each linguistic variable was indicated using a Triangular Fuzzy Number (TFN) with a range from 0 to 100 . Let $\tilde{E}_{i j}^{k}$ indicate the fuzzy performance value of evaluator $k$ toward strategy $i$ under criteria $j$ and the performance of the criteria is represented by the $S$, then,

$\tilde{E}_{i j}^{k}=\left(L \tilde{E}_{i j}^{k}, M \tilde{E}_{i j}^{k}, U \tilde{E}_{i j}^{k}\right), \quad j \in S$

In this study, we used the notion of average value to consolidate the fuzzy judgment value of $\mathrm{m}$ evaluators, i.e.,

$\tilde{E}_{i j}^{k}=(1 / \mathrm{m}) \odot\left(\tilde{E}_{i j}^{1} \oplus \tilde{E}_{i j}^{2} \oplus \cdots \oplus \tilde{E}_{i j}^{m}\right)$

The sign $\odot$ denotes fuzzy multiplication and the sign $\oplus$ denotes fuzzy addition. $\tilde{E}_{i j}^{k}$ is the average fuzzy number from the judgment of the decision-maker. It can be represented using a triangular fuzzy number as follows:

$\tilde{E}_{i j}^{k}=\left(L \tilde{E}_{i j}^{k}, M \tilde{E}_{i j}^{k}, U \tilde{E}_{i j}^{k}\right)$

where,

$$
\begin{aligned}
& \left.L \tilde{E}_{i j}^{k}=(1 / m) \odot\left(\sum_{k=1}^{m} L \tilde{E}_{i j}^{k}\right)\right) \\
& \left.M \tilde{E}_{i j}^{k}=(1 / m) \odot\left(\sum_{k=1}^{m} M \tilde{E}_{i j}^{k}\right)\right) \\
& \left.U \tilde{E}_{i j}^{k}=(1 / m) \odot\left(\sum_{k=1}^{m} U \tilde{E}_{i j}^{k}\right)\right)
\end{aligned}
$$

The preceding end point value can be solved using the method introduced by Buckly (1985) or by 
Chiou and Tzeng. (2002).

\subsubsection{Fuzzy synthetic decision}

The weight of the different criteria and the fuzzy performance value needs to be operated using fuzzy integral techniques to generate the synthetic performance of each strategy within the same dimension.

Furthermore, we have calculated the synthetic performance of each alternative strategy using different $\lambda$ values. Additionally, the fuzzy synthetic performance is conducted by a simple additive weight method assuming the criteria are independent in a fuzzy environment. Since each individual criterion is not completely independent from the others, we use the non-additive fuzzy integral technique to find the synthetic performance of each alternative, and to investigate the order of the synthetic performance of different $\lambda$ values.

The result of fuzzy synthetic decisions reached by each alternative is a fuzzy number. It is therefore the non-fuzzy ranking method for fuzzy numbers that must be employed in order to compare the various strategies. In previous works the procedure of de-fuzzification had involved the location of the best non-fuzzy performance (BNP) value. The methods for defuzzified fuzzy ranking generally include the mean of maximum, center of area (COA), and $\alpha$-cut (Zhao and Govind, 1991; Opricovic and Tzeng, 2003).

We utilize the center of area (COA) method in this paper to rank the order of importance of each strategy. The BNP value for the fuzzy number $\tilde{R}_{i}$ can be found using the following equation:

$$
B N P_{i}=\left[\left(U \tilde{R}_{i}-L \tilde{R}_{i}\right)+\left(M \tilde{R}_{i}-L \tilde{R}_{i}\right)\right] / 3+L \tilde{R}_{i} \quad \forall i
$$

\section{Empirical Study and Discussions}

In order to demonstrate the practicality of our proposed method of enhancing the performance mutual funds, we conducted an empirical study based on a total of 30 valid samples from 12 Taiwanese mutual fund companies and 8 research institutes and universities.

The majority of the respondents were fund managers who are responsible for financial or general management. The mutual fund strategy selection process is examined below.

\subsection{Evaluating the weights of issues}

By using the fuzzy AHP method the weights of the issues and aspects were found and are shown in Table 1.

\subsection{Estimating the performance matrix}

In this study, the evaluators define their individual range for the linguistic variables employed in this study based on their judgments within the range from 0-100. The fuzzy judgment values of different evaluators regarding the same evaluation criteria are averaged. In general, fuzzy addition and multiplication were used to retrieve the average fuzzy numbers for the performance values under each criterion indicated by the evaluators for mutual fund strategy.

Table 1 The weights of issues for evaluating the mutual funds 


\section{Market timing}

(0.236 0.4270 .719 )

0.461

the ratio of fund market share

(0.114 0.1970 .359 )

0.223

the return of market

(0.263 0.4370 .729 )

0.476

riskless interest rate

(0.130 0.2420 .432$)$

0.268

flowing of cash

(0.066 0.124 0.226)

0.139

Stock selection ability

(0.218 0.3530 .592$)$

0.388

$\mathrm{P} / \mathrm{E}$ ratio

(0.119 0.2110 .368$)$

0.232

net value/market value

(0.081 0.1430 .257 )

0.160

cash flowing/market value

(0.039 0.0620 .110$)$

0.070

net value

(0.097 0.1720 .323$)$

0.197

risk premium

(0.235 0.4120 .694$)$

0.571

\section{Fund size}

(0.090 0.1430 .244$)$

0.159

the market share of mutual fund

(0.207 0.3230 .522$)$

0.351

the growth rate of mutual fund scale

(0.087 0.1290 .218$)$

0.145

dividend yield of mutual fund

(0.335 0.5480 .851$)$

0.578

Team work

(0.049 0.076 0.133)

0.086

number of researcher

(0.130 0.2690 .452$)$

0.284

education of fund manager

(l) $\left.\begin{array}{lll}0.081 & 0.138 & 0.270\end{array}\right)$

0.163

known of fund manager

(0.253 0.4390 .776$)$

0.489

turnover rate of mutual fund

(0.095 0.1540 .292$)$

0.180

Table 2 The evaluation results of mutual fund strategy

\section{Mutual fund strategy ranking}

$\begin{array}{ll}\text { SAW: } & \mathrm{S} 4 \succ \mathrm{S} 3 \succ \mathrm{S} 2 \succ \mathrm{S} 1 \succ \mathrm{S} 5 \\ \lambda=-1,0.5: & \mathrm{S} 2 \succ \mathrm{S} 4 \succ \mathrm{S} 3 \succ \mathrm{S} 1 \succ \mathrm{S} 5 \\ \lambda=0,1,3,5, \ldots, 100 ; & \mathrm{S} 4 \succ \mathrm{S} 3 \succ \mathrm{S} 2 \succ \mathrm{S} 1 \succ \mathrm{S} 5 \\ \lambda=150 ; & \mathrm{S} 4 \succ \mathrm{S} 2 \succ \mathrm{S} 3 \succ \mathrm{S} 1 \succ \mathrm{S} 5 \\ \lambda=200 ; & \mathrm{S} 4 \succ \mathrm{S} 1 \succ \mathrm{S} 2 \succ \mathrm{S} 3 \succ \mathrm{S} 5\end{array}$

where: S1: Asset Allocation style; S2: Aggressive Growth style; S3: Equity Income style; S4: Growth style; S5: Growth Income style. 
Table 3. The synthetic performance of mutual fund strategy

\begin{tabular}{|c|c|c|c|c|c|c|c|c|c|}
\hline \multicolumn{10}{|l|}{$\lambda$} \\
\hline & -1.00 & -0.50 & 0.00 & 1.00 & 3.00 & 5.00 & 10.00 & 40.00 & 100.00150 .00 200.00. SAW \\
\hline Aa & 385.25 & 527.97 & 299.72 & 298.89 & 297.82 & 297.13 & 296.07 & 293.73 & 3292.47291 .55291 .08303 .47 \\
\hline Ag & 606.77 & 971.22 & 310.88 & 309.76 & 307.63 & 306.00 & 303.26 & 297.12 & 294.00291 .88290 .87317 .37 \\
\hline $\mathrm{Ei}$ & 459.00 & 672.52 & 312.89 & 311.82 & 309.50 & 307.67 & 304.55 & 297.57 & 7294.42291 .70290 .58318 .77 \\
\hline G & 553.23 & 856.70 & 314.68 & 313.72 & 311.79 & 310.25 & 307.59 & 301.30 & 297.94295 .69294 .59320 .86 \\
\hline Gi & 351.17 & 441.07 & 275.72 & 273.92 & 271.77 & 270.47 & 268.59 & 265.26 & 263.92263 .10262 .75277 .89 \\
\hline
\end{tabular}

\subsection{Evaluation and prioritization of the mutual fund strategy}

The empirical evidence in the paper indicates that the weight of criteria such as market timing (0.461), stock selection ability (0.388), fund size (0.159) and team work (0.086). So the market timing was the most important factors to influence the performance of mutual fund, next was the stock selection ability. Some econometric methodology is developed to simultaneously estimate the magnitudes of these portfolio performance evaluation measures. The results show that mutual fund managers are on average with positive security selection and negative market timing ability. It means that mutual fund managers are on average better with selectivity ability than with market timing ability. Therefore, the mutual fund managers should enhance the ability of market timing, the performance of mutual fund can be better.

The fuzzy synthetic performance of each alternative using different $\lambda$ is as shown in Table 2 and Table 3. In table 2, our empirical results show that when $\lambda<0$, the results show that S2 "Aggressive Growth style" was the most important strategy. And S4, "Growth style” was selected as the second most important strategy. When $\lambda \geq 0$, the results show that $\mathrm{S} 4$ "Growth style" was the most important strategy regardless of the variation of $\lambda$. And S3, "Equity Income style" was selected as the second most important strategy. When $\lambda>150$, S2 replaces S3, becoming the number 2 ranking. But $\lambda \geq 200$, S1 replaces S2 and S3, becoming the number 2 ranking. We can infer that the asset allocation style more efficiency when multiplicative effect exists.

From Table 3, we know that when $\lambda$ was bigger, the synthetic performance was smaller. So these results implied that mutual funds have no multiplicative effect. Therefore, from investment style average shows that the aggressive growth style has the largest maximum performance, but it also has the largest deviation in performance. In other word, the more aggressive the funds are, the more volatility of the fund performance will be. However, the growth income style has the smallest expected performance. And next was asset allocation style.

\subsection{Discussions and managerial implications}

This study focuses on providing a mutual fund strategy model for the companies of mutual funds managers so that they may be successful in their decision-making. Our empirical study demonstrates the validity of this model. In this study, the mutual funds strategy stems from four aspects: Market timing, Stock selection ability, Fund size as well as team work. The related issues, evaluation criteria and strategies are defined in this research. 
Mutual funds managers have difficulty in utilizing the proper strategy. The major reasons are the fuzzy and dynamic environment and numerous criteria that they are facing. Managers are hence overwhelmed by this vague scenario and do not make proper decisions or allocate resources efficiently. The hierarchical model guides the manager how to select the style of mutual fund in the uncertainty environment. With the help of this model, managers can employ different experts to conduct the same proposed procedures and select the best investment alternative. The subjective judgment and risks of making wrong decisions is then minimized. In addition, this method can be applied to solve different kinds of problems by modifying the constructs of the hierarchy trees and finding the appropriate solution.

\section{Conclusion}

The mutual fund is moving rapidly towards financial market development in response to increasing market demand. The traditional quantity method does not solve the complex problems of mutual fund development. Therefore, what is needed is a useful and applicable strategy that addresses the issues of developing mutual funds. This study addressed this issue in a more rational and objective fashion by using a FMCDM model to achieve this goal.

Few studies have addressed mutual fund -related strategy planning. Providing that this is a first attempt to formally model the formulation process for a mutual fund strategy using FMCDM, we have the confidence that the analysis here is a significant theoretical contribution to the literature, and will help to establish groundwork for future research. Even though we are dedicated to setting up the model as completely as possible, there are additional criteria and methods that could be adopted and added in future research. In the meantime, we should also begin to investigate how to execute several strategies simultaneously in order to achieve the best performance of mutual fund under the constraint of resources.

\section{References}

Bhattacharya, S., and Pfleiderer, P. (1983) “A note on performance evaluation.”

Bellman, R.E. and Zadeh, L.A. (1970) “Decision-making in a fuzzy environment”, Management Science Vol.17, No.4, pp.141-146.

Buckley, J.J. (1985) “Ranking alternatives using fuzzy numbers”, Fuzzy Sets and Systems Vol.15, No.1, pp.21-31.

Connor, G., and R. Korajczyk (1991) “Performance measure with the arbitrage pricing theory.”Journal of financial Economics 15, 373-394.Treynor, J. L., and Technical Report 714. Stanford, Calif,: Stanford University, Graduate School of Business.

Lee, C. F., and S. Rahman (1990) "Market timing, selectivity, and mutual fund performance: An empirical investigation.” Journal of Business, 63, 261-78. 
Chen Y.W. and Tzeng, G.H. (2001) “Using fuzzy integral for evaluating subjectively perceived travel cost in a traffic assignment model”, European Journal of Operation Research Vol.130, No.3, pp.653-664.

Chiou, H.K. and Tzeng G.H. (2002) “Fuzzy multicriteria decision making approach to analysis and evaluation of green engineering for industry”, Environmental Management, Vol.30, No.6, pp.816-830

Fama, E. F. (1972) “Components of investment performance.” Journal of Finance 27, 551-67.

Henriksson, R. D. (1984) “Market timing and mutual fund performance : An empirical investigation.” Journal of Business 57, 73-96.

H. Markowitz, (1952) “Portfolio Selection”, Journal of Finance Vol.7, No.1, pp. 77-91.

J. Watada, (2001) “Fuzzy Portfolio Model for Decision Making in Investment,” Dynamical Aspects in Fuzzy Decision Making, pp. 141-162.

Keeney R. and Faiffa, H. (1976) “Decisions with multiple objective preferences and value tradeoffs,” Cambridge University Press.

Klir G.J. and Folger, T.A. (1998) “Fuzzy sets, uncertainty, and information.” Prentice Hall, Englewood Cliffs.

Lehmann, B. N., and D. M. Modest (1987) "Mutual fund performance evaluation: A comparison of benchmarks and benchmark comparisons." Journal of Finance, 42,233-265.

Murofushi, T. and Sugeno, M. (1989) “An Interpretation of Fuzzy Measures and the Choquet integral as an Integral with Respect to a Fuzzy Measure”, Fuzzy Sets and Systems Vol.29, No.2, pp. 201-227.

Murofushi, T. and Sugeno, M. (1991) “A Theory of Fuzzy Measures Representations, the Choquet Integral and Unll Sets”, Journal of Mathematical Analysis and Application Vol.159, No.2, pp.532-549.

Opricovic, S. and Tzeng, G.H. (2003) “Defuzzification within a multicriteria decision model”, International Journal of Uncertainty, Fuzziness and Knowledge-Based Systems, Vol.11, No.5 (Forthcoming).

Sugeno, M. and Terano, T. (1997) “A model of learning based on fuzzy information”, Kybernetes Vol.6, No.2, pp.157-166.

Sugeno, M. (1974) “Theory of fuzzy integrals and ITS applications,” Ph.D. Dissertation. Tokyo Institute of Technology.

Sugeno, M. and Kwon, S.H. (1995) “A clusterwise regression-type model for subjective evaluation”, Journal of Japan Society for Fuzzy Theory and Systems Vol.7, No.2, pp. 291-310.

Tang, M.T., G.H. Tzeng, Wang, S.W. (1999) “A hierarchy fuzzy MCDM method for studying electronic marketing strategies in the information service industry”,.Journal of International Information Management Vol 8, No.1, pp. 1-22. 
Treynor, J. L., and Mazuy, K. K. (1966) “Can mutual funds outguess the market ?” Harvard Business Review, 44, 131-36.

Tsaur, S.H. and Tzeng, G.H. and Wang, G.C.(1997) “The application of AHP and fuzzy MCDM on the evaluation study of tourist risk", Annals of Tourism Research, Vol.24, No.4, pp.796-812.

T. Watanabe, K. Oda and J. Watada, (1999) “ Hierarchical Decision Making of Strategic Investment” International Journal on Fuzziness, Uncertainty, and Knowledge-Based Reasoning (IJUFKS), Vol.7,No.4,

Tzeng, G.H. (1977) “A study on the PATTERN method for the decision process in the public system”, Japan Journal of Behaviourmetrics Vol.4, No.2, pp.29-44.

Watada, J.,(1999) “Soft-Computing Approaches to Portfolio Selection Problems”, Working Paper.

Yu, P.L. (1985) Multiple criteria decision making: concepts, techniques and extensions, Plenum Press, New York.

Yu, P.L. (1990) Forming winning strategies: an integrated theory of habitual domains, Springer-Verlag, Berlin, Heidelberg, New York.

Yu, P.L. (1995) Habitual domain, Highwater Editions.

Zhao R. and Govind, R. (1991) “Algebraic characteristic of extended fuzzy numbers”, Information Science Vol.54, No.1, pp.103-130 